\title{
Bdellovibrio bacteriovorus HD100, a predator of Gram-negative bacteria, benefits energetically from Staphylococcus aureus biofilms without predation
}

\author{
Hansol $\operatorname{Im}^{1} \cdot$ Mohammed Dwidar $\mathbb{D}^{2} \cdot$ Robert J. Mitchell ${ }^{1}$
}

Received: 26 January 2018 / Revised: 11 April 2018 / Accepted: 17 April 2018 / Published online: 30 May 2018

(c) International Society for Microbial Ecology 2018

\begin{abstract}
Bdellovibrio bacteriovorus HD100 is a predatory bacterium which lives by invading the periplasm of Gram-negative bacteria and consuming them from within. Although B. bacteriovorus HD100 attacks only Gram-negative bacterial strains, our work here shows attack-phase predatory cells also benefit from interacting with Gram-positive biofilms. Using Staphylococcus aureus biofilms, we show this predator degrades the biofilm matrix, obtains nutrients and uses these to produce and secrete proteolytic enzymes to continue this process. When exposed to $S$. aureus biofilms, the transcriptome of B. bacteriovorus HD100 was analogous to that seen when present intraperiplasmically, suggesting it is responding similarly as when in a prey. Moreover, two of the induced proteases (Bd2269 and Bd2692) were purified and their activities against $S$. aureus biofilms verified. In addition, B. bacteriovorus HD100 gained several clear benefits from its interactions with $S$. aureus biofilms, including increased ATP pools and improved downstream predatory activities when provided prey.
\end{abstract}

Bdellovibrio bacteriovorus is a Gram-negative obligate predatory bacterium which lives by attacking other Gramnegative bacteria [1], including a very wide range of human pathogens $[2,3]$ but does not predate on Gram-positive bacterial strains [2, 4-7]. This predator enters the periplasm of its prey, where it hydrolyzes and consumes the prey cell components, grows and septates before lysing the prey and proceeding to attack another. Although a previous study showed the addition of glutamate or glutamate and other

These authors contributed equally: Hansol Im and Mohammed Dwidar

Electronic supplementary material The online version of this article (https://doi.org/10.1038/s41396-018-0154-5) contains supplementary material, which is available to authorized users.

Mohammed Dwidar medwidar2002@gmail.com

$\triangle$ Robert J. Mitchell esgott@unist.ac.kr

1 School of Life Sciences, Ulsan National Institute of Science and Technology (UNIST), 50 UNIST-gil, Ulsan 44919, Republic of Korea

2 Nucleic Acid Chemistry and Engineering Unit, Okinawa Institute of Science and Technology (OIST), 1919-1 Tancha, Onna-son, Kunigami-gun, Okinawa, Japan 904-0495 amino acids prevented loss of viability [8], it has long been held that this predator is dependent on prey cells for both its replication and as a source of chemical energy. In addition to attacking planktonic prey, this predator is quite capable of reducing Gram-negative biofilm populations [2, 9, 10], even when two preys were present together [2]. In nature, however, B. bacteriovorus is thought to be mainly associated with biofilms $[11,12]$ and, as such, encounters nonprey Gram-positive strains within these biofilms.

To evaluate how B. bacteriovorus HD100 responds to Gram-positive biofilms, therefore, Staphylococcus aureus was selected since it is a non-prey bacterium [5] and a well-known human pathogen. Figure 1a illustrates a basic characteristic of predatory bacteria, namely that they only prey on Gramnegative bacteria, leading to an increase in their numbers. We found that when added to pre-formed biofilms, the predatory culture dispersed $S$. aureus biofilms (Fig. 1b) to the same degree as prey, i.e., Salmonella enterica, biofilms based upon crystal violet (CV) staining (Fig. 1c). Figure 1d shows that, while the CV results were similar, the $S$. enterica biofilm population was reduced by more than $1-\log$ while that of $S$. aureus was reduced by only $59 \%$. Though mild in comparison, this loss was significant, proving B. bacteriovorus HD100's ability to disperse $S$. aureus biofilms.

Given the susceptibility of $S$. aureus biofilms to proteases [5, 13-15], we hypothesized residual proteases from 
A)

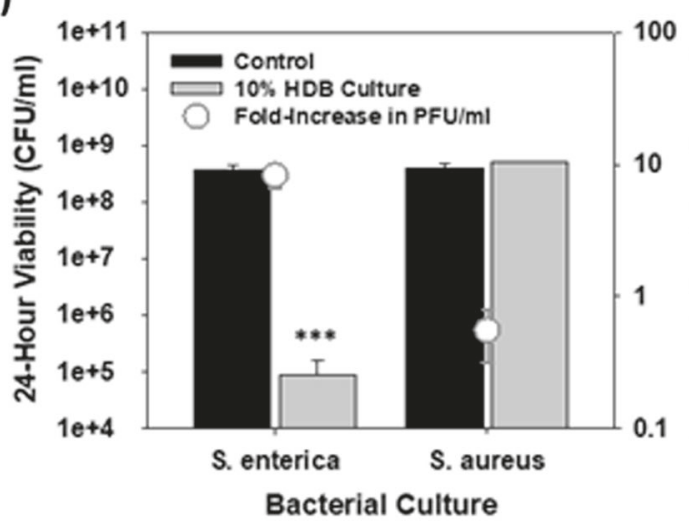

C)

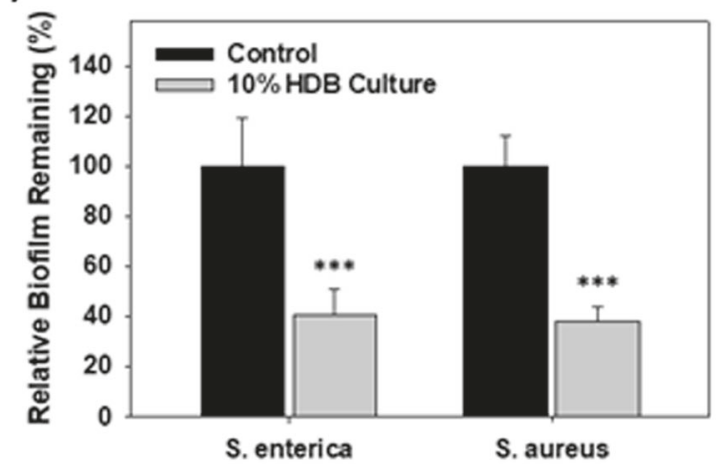

Bacterial Culture
B)
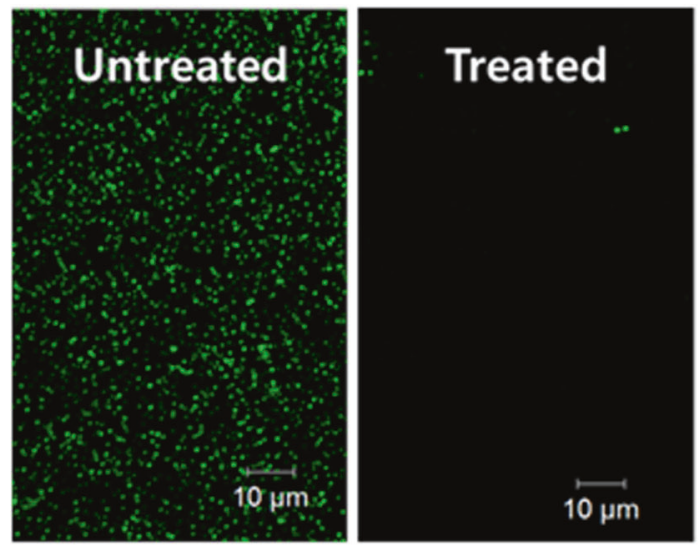

D)
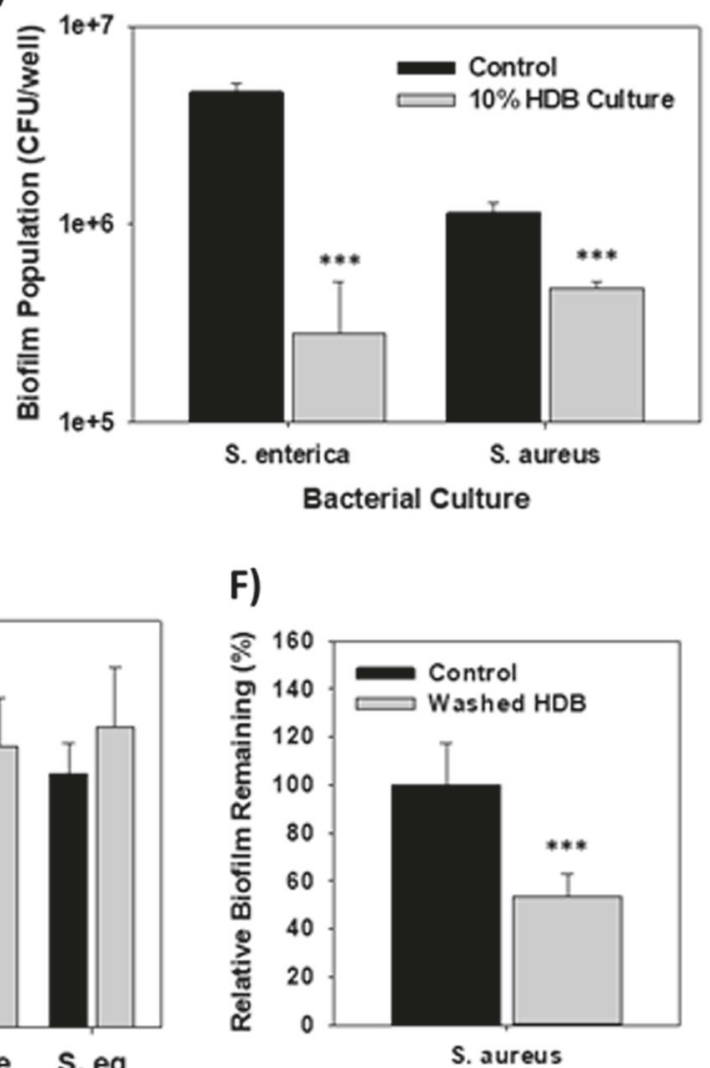

E)

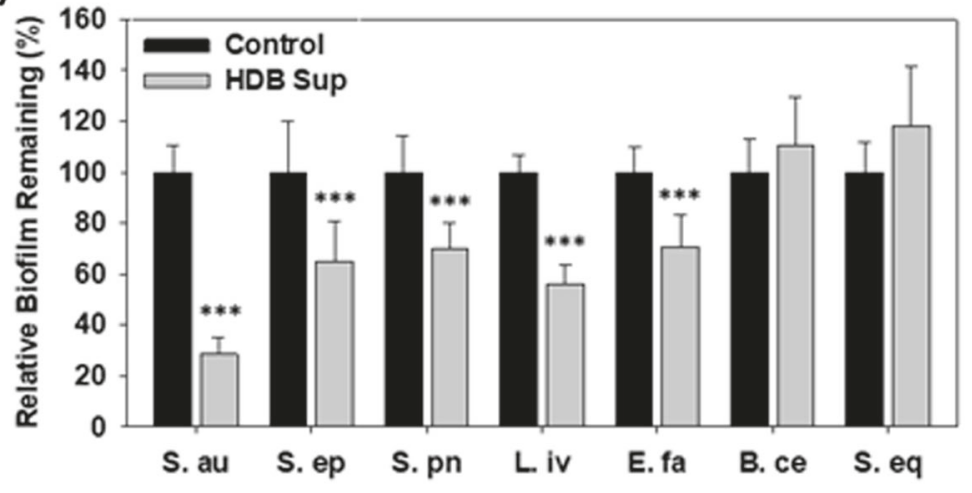

F)

Bacterial Culture the previous predation cycle, i.e., when culturing this predator for these tests, were responsible for the dispersions seen. The presence of proteases in the cell-free spent media (HDB Sup) was confirmed using the Azocoll assay, with average residual activities equivalent to $3.2 \pm$ $1.3 \mathrm{ng} / \mathrm{ml}$ proteinase $\mathrm{K}$. This was sufficient, even when diluted tenfold, to significantly disperse several different Gram-positive biofilms (Fig. 1e). The use of proteinase $\mathrm{K}$ led to similar levels of dispersion while the addition of AEBSF, a potent inhibitor of serine proteases, reduced the activity of the predatory supernatants (Figures S1 and S2). One interesting finding was the treatment of $S$. enterica biofilms with proteases actually increased the biomass (Figure S2). This increase is thought to be due to loss of the flagella, which causes the cells to adhere to the bottom of the wells as described by Teplitski et al [16]. Similarly, the differences seen between the individual Gram-positive biofilms is likely due to variations in their EPS content, a concept that should be studied in a future work. 
Fig. 1 B. bacteriovorus disperses Gram-positive biofilms. a S. aureus is not predated upon by $B$. bacteriovorus HD100. Cultures of $S$. enterica or $S$. aureus were incubated in HEPES buffer alone (Control) or in HEPES containing B. bacteriovorus HD100 (10\% HDB Culture). The 24-h viability of each bacterium was then determined. For $B$. bacteriovorus HD100, the results are presented as the fold-increase in the predatory population, illustrating that $S$. enterica is a prey but $S$. aureus is not. ${ }^{* * *} P<0.001$. $(n=3)$. b Confocal images of the $S$. aureus biofilm showing its removal when treated with $B$. bacteriovorus HD100 in HEPES buffer. The untreated sample was incubated in sterile HEPES buffer. After treatment, the cells were stained with Syto-9 to visualize them. c B. bacteriovorus HD100 removes both $S$. enterica and $S$. aureus biofilms. Biofilms of each strain alone in 96well plates were treated for $24 \mathrm{~h}$ with HEPES buffer alone (Control) or with HEPES containing B. bacteriovorus HD100 (10\% HDB Culture). The results are presented as the relative biofilm biomass based upon crystal violet staining. $* * * P<0.001$. $(n=27)$. d $B$. bacteriovorus HD100 reduced $S$. aureus biofilm populations. The bacterial populations remaining after treatment with $B$. bacteriovorus $\mathrm{HD} 100$ as in $\mathbf{c}$ were determined, proving $S$. aureus is being removed. $* * * P<0.001$. $(n=3)$. e Dispersal of various Gram-positive biofilms by the cell-free predatory supernatant (HDB Sup). HDBsup was added at $10 \%$ (v:v) to preformed biofilms of each bacterium. The strains used in this figure were S. au (Staphylococcus aureus KACC 10768), S. ep (Staphylococcus epidermidis KACC 13234), S. pn (Streptococcus pneumoniae KACC 15313), L. iv (Listeria ivanovii subsp. ivanovii KACC 10767), E. fa (Enterococcus faecalis KACC 11304), B. ce (Bacillus cereus KACC 1000), and S. eq (Streptococcus equinus KACC 13808). The biomass remaining was determined after $24 \mathrm{~h} . * * * P<0.001$. $(n=10)$. f $B$. bacteriovorus HD100 cells that were pelleted and washed to remove any extracellular proteases still dispersed the $S$. aureus biofilms. $* * * P<0.001$. $(n=10)$. The protocols used for these experiments are provided within the Supplemental Information and Data

In subsequent experiments, we found $S$. aureus biofilms were still dispersed even after the B. bacteriovorus HD100 cells were pelleted and washed to remove the residual secreted proteases (Fig. If and S3), implying de novo protease expression also occurred. As shown in Figure S3, this was also true for other $S$. aureus biofilms, demonstrating this activity is not limited to a single strain of $S$. aureus. Figure $\mathrm{S} 4$ demonstrates the proteolytic activities were from the predatory strain, and not the $S$. aureus biofilms, as the resulting proteolytic activity levels were nearly identical and the level of dispersion was comparable regardless if the biofilm was viable or UV-killed.

We next studied how B. bacteriovorus HD100 responds to $S$. aureus biofilms by sequencing the total cellular RNA. As shown in Figure 2a, the transcriptomic responses during the exposure are highly reminiscent of those seen both during intraperiplasmic (IP) growth [17] and when B. bacteriovorus HD100 was incubated in nutrient broth (NB) [18]. One striking difference between the $S$ aureus biofilminduced transcriptomic responses and the other conditions was EggNOG (Evolutionary genealogy of genes: Nonsupervised Orthologous Groups) category "N" (Fig. 2a), which includes the genes related with cell motility. Whereas most of the genes included in this category were downregulated in the NB-treated and IP predatory cultures, the majority were up-regulated when $B$. bacteriovorus HD100 was exposed to $S$. aureus biofilms. Of the 38 EggNOG category "N" genes up-regulated in this study, 35 are annotated as being involved in flagellar biosynthesis and were located within eight clusters or operons throughout the chromosome. The other three are annotated as a transporter related with gliding motility (Bd1025), a chemotaxis protein (CheX, Bd1823) and a hypothetical protein located within a flagellar operon (Bd3396). The differences between the three studies are thought to be due to the nature of the predator and its environments; the release of amino acids from the $S$. aureus biofilm through the induced proteolytic activities may act as a chemoattractant for swimming predator cells, as demonstrated by LaMarre et al. [19] in their study with Bacteriovorax stolpii UKi2. In contrast, IP growth requires little or no movement while the media used by Dwidar et al. [18] was homogeneous, precluding the need for the flagella or chemotactic responses. Likewise, the predators exposed to the $S$. aureus biofilms tended to have fewer EggNOG category "D" genes upregulated than the other published conditions. The reason for this is the variability in the gene expression levels; although many genes had average expression levels that were upregulated several-fold, their values were not significant based upon the Student's $t$-test. Consequently, these were categorized as being "No Significant Change or Silent".

When viewed alongside the findings of Dwidar et al. [18], where B. bacteriovorus HD100 altered its transcriptome and secreted proteases in response to nutrients in the form of nutrient broth, the above results imply B. bacteriovorus HD100 also responds to $S$. aureus biofilms as if they were a source of amino acids and secretes proteases in reply. This was confirmed by both the transcriptomic data, where the expression levels for several different serine protease genes were elevated when B. bacteriovorus HD100 was exposed to the $S$. aureus biofilms for $4 \mathrm{~h}$ (Figure S5), and the increased extracellular proteolytic activities, which paralleled those seen within tests with NB (Fig. 2b). Two of the proteases ( $\mathrm{Bd} 2269$ and $\mathrm{Bd} 2692)$ induced in B. bacteriovorus HD100 by the $S$. aureus biofilms (Figure S5) were cloned, then expressed and purified (Figures S6 and S7). When tested, we found $S$. aureus biofilms were susceptible to both (Fig. 2c). Their clear activities against $S$. aureus biofilms, alongside their expression patterns, imply these two proteases are likely involved in the dispersals seen above and that they provide this predatory bacterium with a source of amino acids.

These amino acids not only provided $B$. bacteriovorus HD100 with the necessary components to express and secrete proteases but also provided it with a source of energy. This is illustrated in Figure $2 \mathrm{~d}$ where $B$. bacteriovorus HD100's ATP pool was significantly higher (1.7fold) after an exposure to the $S$. aureus biofilms when 
A)

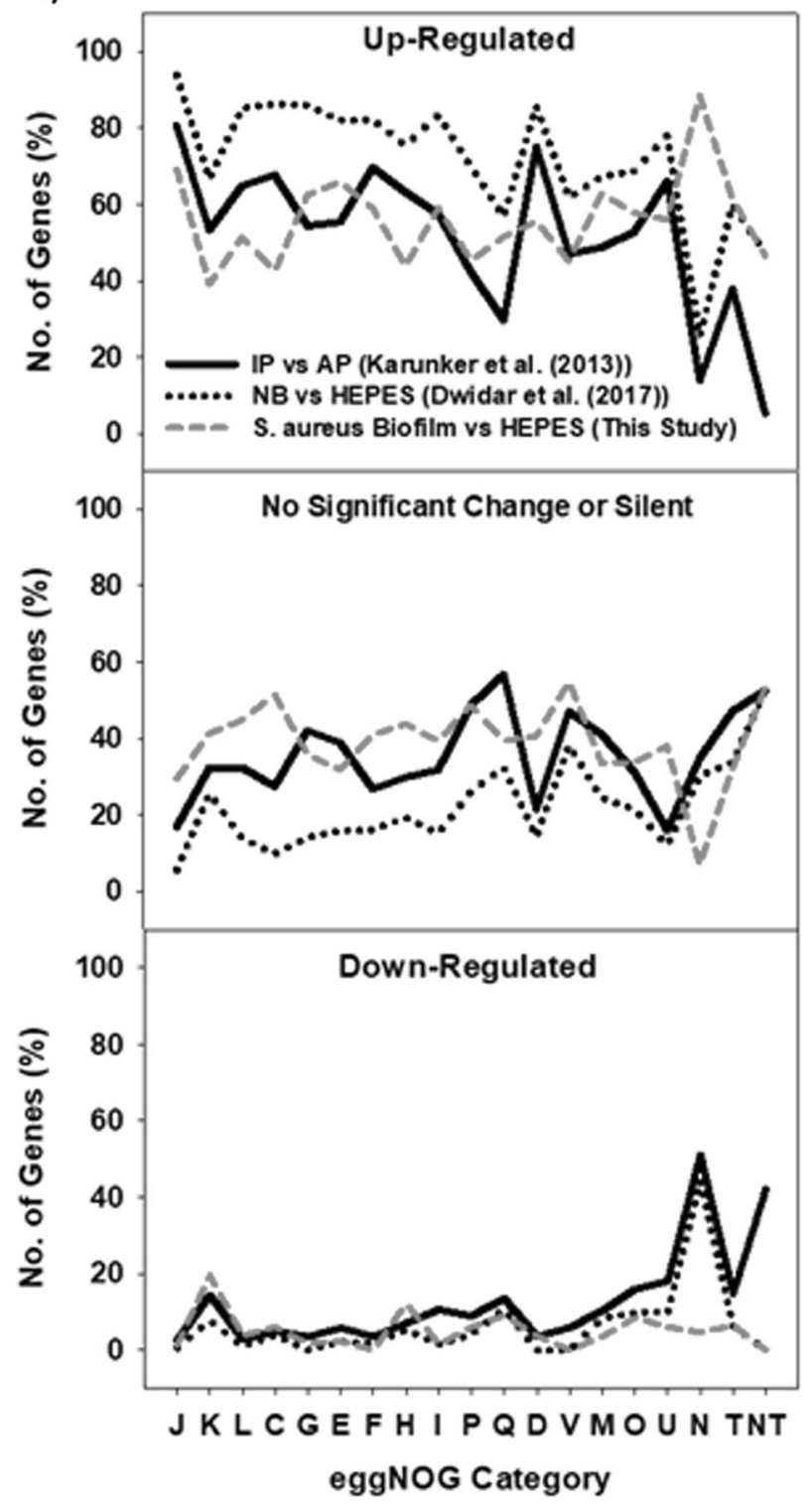

B)

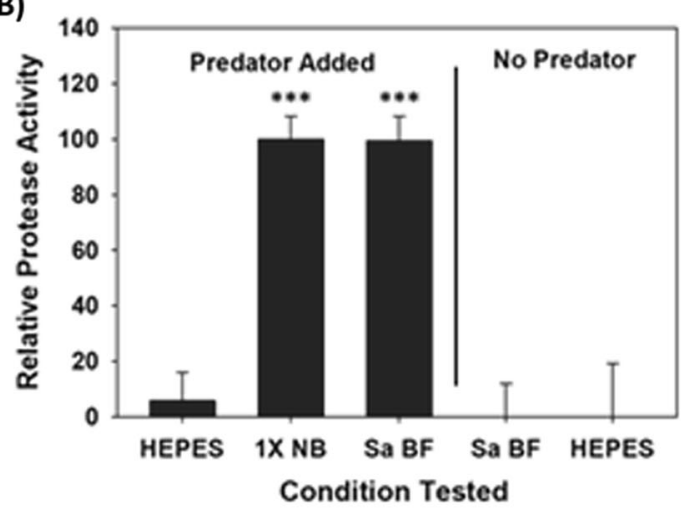

C)
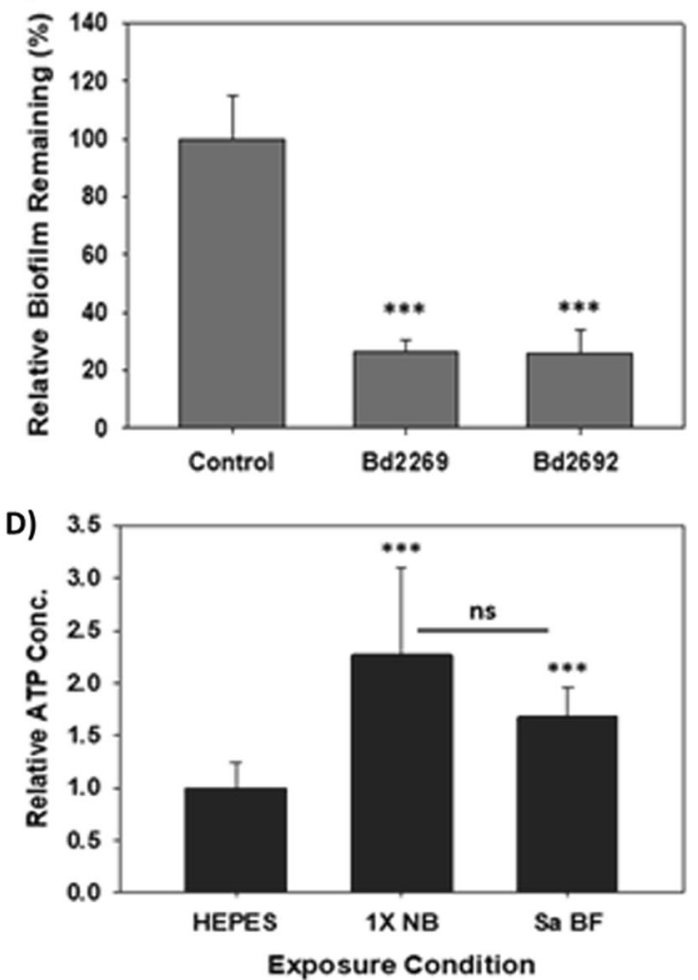

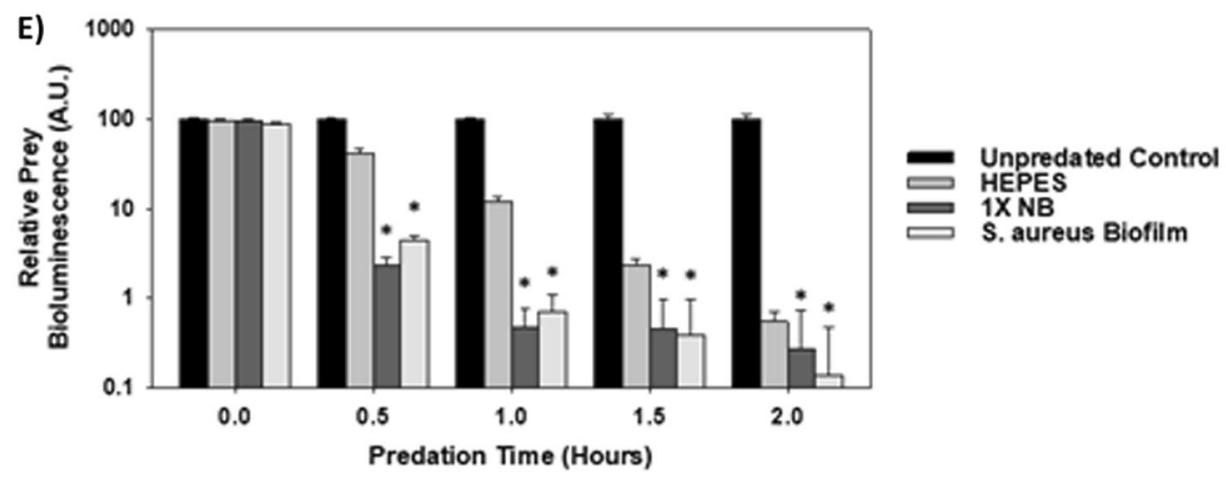

compared to predatory cells incubated for the same amount of time in HEPES. The higher ATP levels were slightly less than but statistically comparable to those seen when $B$. bacteriovorus HD100 was provided nutrients in the form of
NB medium. The higher energy content in both predatory cultures correlated with better predatory activities downstream, as illustrated in Figure 2e. In this assay, the prey was a bioluminescent strain of $E$. coli and the relative loss in 
Fig. 2 Extracellular nutrients and biofilms induce the secretion of proteases by attack-phase host-dependent $B$. bacteriovorus. a EggNOG-based analysis of $B$. bacteriovorus HD100 gene expression patterns when exposed to $S$. aureus biofilms or HEPES buffer alone. This figure shows the results of this study juxtaposed against previously published data, i.e., an NB/HEPES transition [18] and a GP3/ AP transition [17]. Excluding the NT group, any genes located within more than one category were excluded from the analyses. In addition, only those genes showing more than 1.5 -fold change in their expression (with $P<0.05$ ) were considered. For the GP3/AP transition [17], the authors provided Bonferroni values instead of $p$-values. J: Translation, ribosomal structure and biogenesis, $\mathrm{K}$ : Transcription, L: Replication, recombination and repair, C: Energy production and conversion, G: Carbohydrate transport and metabolism, E: Amino acid transport and metabolism, F: Nucleotide transport and metabolism, H: Coenzyme transport and metabolism, I: Lipid transport and metabolism, P: Inorganic ion transport and metabolism, Q: Secondary metabolites biosynthesis, transport and catabolism, D: Cell cycle control, cell division, chromosome partitioning, V: Defense mechanisms, M: Cell wall/membrane/envelope biogenesis, O: Posttranslational modification, protein turnover, chaperones, U: Intracellular trafficking, secretion, and vesicular transport, N: Cell motility, T: Signal transduction mechanisms, NT: Both cell motility and signal transduction mechanisms. Portions of this figure were reprinted with permission from ref. [18]. b $S$. aureus biofilms and NB media induce similar levels of protease secretion from B. bacteriovorus. Washed attackphase $B$. bacteriovorus were exposed for $24 \mathrm{~h}$ to HEPES buffer alone (HEPES), NB media $(1 \times \mathrm{NB})$ or $S$. aureus biofilms in HEPES buffer (Sa BF) within 96-well plates. The control wells had only HEPES alone (HEPES) or $S$. aureus biofilms alone (Sa BF), i.e., without the predator added. The relative protease activity (based on $1 \times \mathrm{NB}$ ) of each sample was measured after $24 \mathrm{~h}$ and compared with that of the predator in HEPES. $* * * P<0.001$. $(n=6)$. c Purified B. bacteriovorus proteases are capable of removing $S$. aureus biofilms. The $S$. aureus biofilms were treated for $24 \mathrm{~h}$ with HEPES buffer alone or supplemented with the individual proteases, Bd2269 or Bd2692, which were added at an activity equivalent of $1 \mathrm{ng}$-Proteinase $\mathrm{K} / \mathrm{ml}$ based upon the Azocoll protease assay. $* * * P<0.001 .(n=10)$. d Incubation of $B$. bacteriovorus HD100 with NB media or $S$. aureus biofilms increases the predatory cellular ATP pools. Washed attack-phase B. bacteriovorus were exposed for $6 \mathrm{~h}$ to HEPES buffer alone, $1 \times \mathrm{NB}$ or $S$. aureus biofilms in HEPES buffer. The cellular ATP content was determined afterwards, showing the significantly higher ATP pools in the predatory cells incubated in NB media or with the non-prey biofilms. $* * * P<0.001 ; \mathrm{ns}=$ not significant. $(n=3)$. e Incubation with $S$. aureus biofilms improves the predatory activity against $E$. coli. The activity of the predators in $\mathbf{d}$ were measured using a bioluminescent $E$. coli prey as described previously [20]. The results show that the predatory activity of $B$. bacteriovorus cells exposed to the $S$. aureus biofilms are significantly better than those exposed to HEPES alone. ${ }^{*} P<0.001$. $(n=28)$. The protocols used for these experiments are provided within the Supplemental Information and Data

bioluminescence was used to compare the predatory activities [20]. The faster and more significant loss in bioluminescence seen with the NB- and S. aureus biofilm-incubated $B$. bacteriovorus HD100 cells show these cultures were more active than those incubated in HEPES buffer.

To date, this predator was thought to be dependent upon Gram-negative prey cells as both a source of chemical energy and for its replication, restricting many of the studies published to only those interactions. The findings presented here implicate that bdellovibrios may have an even greater impact on bacterial communities than previously thought and that their interactions within nature are not limited to only Gram-negative strains and biofilms. Although $B$. bacteriovorus does not predate on Gram-positive bacterial strains, despite one study allegedly claiming otherwise [21], at present very little is known about how this predator responds to non-prey strains and, in particular, their biofilms. Here, we demonstrate B. bacteriovorus HD100 benefits from such interactions, obtaining amino acids from non-prey biofilms and using them to produce and secrete proteases as well as augment their ATP pools and predatory activities. These activities in no manner whatsoever suggest predation is occurring, as illustrated by the fact that the predatory numbers do not increase when provided $S$. aureus (Fig. 1a), but suggest a hitherto unknown aspect on how predatory bacteria inherently survive and thrive within natural environments, particularly in niches where Grampositive, non-prey cells are present.

Acknowledgements Funding for this research was sponsored by the National Research Foundation of Korea within the General Research Program (Grant No. 2016R1D1A1A09919912). We thank them for the financial support. The following reagent was provided by the Network on Antimicrobial Resistance in Staphylococcus aureus (NARSA) for distribution by BEI Resources, NIAID, NIH: Staphylococcus aureus subsp. aureus Strain JE2 (NR-46543)

Author contributions MD and $\mathrm{HI}$ designed and carried out the experiments. RJM supervised the experimental work. MD, HI, and RJM evaluated the data. MD, HI, and RJM wrote the manuscript.

\section{Compliance with ethical standards}

Conflict of interest The authors declare that they have no conflict of interest.

\section{References}

1. Dwidar M, Monnappa AK, Mitchell RJ. The dual probiotic and antibiotic nature of Bdellovibrio bacteriovorus. BMB Rep. 2012;45:71-78.

2. Dashiff A, Junka RA, Libera M, Kadouri DE. Predation of human pathogens by the predatory bacteria Micavibrio aeruginosavorus and Bdellovibrio bacteriovorus. J Appl Microbiol. 2011;110:431-44.

3. Dashiff A, Kadouri DE. Predation of oral pathogens by Bdellovibrio bacteriovorus 109J. Mol Oral Microbiol. 2011;26:19-34.

4. Im H, Choi SY, Son S, Mitchell RJ. Combined application of bacterial predation and violacein to kill polymicrobial pathogenic communities. Sci Rep. 2017;7:14415.

5. Monnappa AK, Dwidar M, Seo JK, Hur JH, Mitchell RJ. Bdellovibrio bacteriovorus inhibits Staphylococcus aureus biofilm formation and invasion into human epithelial cells. Sci Rep. 2014;4:3811. https://doi.org/10.1038/Srep03811.

6. Chu WH, Zhu W. Isolation of Bdellovibrio as biological therapeutic agents used for the treatment of Aeromonas hydrophila infection in fish. Zoonoses Public Health. 2010;57:258-64. 
7. Stolp H, Starr MP. Bdellovibrio bacteriovorus Gen. Et Sp. N., a predatory, ectoparasitic, and bacteriolytic microorganism. Antonie Leeuwenhoek. 1963;29:217-48.

8. Hespell RB, Rosson RA, Thomashow MF, Rittenberg SC. Respiration of Bdellovibrio bacteriovorus strain 109J and its energy substrates for intraperiplasmic growth. J Bacteriol. 1973;113:1280-88.

9. Chanyi RM, Koval SF, Brooke JS. Stenotrophomonas maltophilia biofilm reduction by Bdellovibrio exovorus. Env Microbiol Rep. 2016;8:343-51.

10. Kadouri D, O'Toole GA. Susceptibility of biofilms to Bdellovibrio bacteriovorus attack. Appl Environ Microb. 2005;71:4044-51.

11. Kelley JI, Turng B, Williams HN, Baer ML. Effects of temperature, salinity, and substrate on the colonization of surfaces in situ by aquatic bdellovibrios. Appl Environ Microbiol. 1997;63:84-90.

12. Williams HN, Schoeffield AJ, Guether D, Kelley J, Shah D, Falkler WA. Recovery of bdellovibrios from submerged surfaces and other aquatic habitats. Microb Ecol. 1995;29:39-48.

13. Iwase T, Uehara Y, Shinji H, Tajima A, Seo H, Takada K, et al. Staphylococcus epidermidis Esp inhibits Staphylococcus aureus biofilm formation and nasal colonization. Nature. 2010;465:346-U100.

14. Lauderdale KJ, Malone CL, Boles BR, Morcuende J, Horswill AR. Biofilm dispersal of community-associated methicillin- resistant Staphylococcus aureus on orthopedic implant material. J Orthop Res. 2010;28:55-61.

15. Elchinger PH, Delattre C, Faure S, Roy O, Badel S, Bernardi T, et al. Effect of proteases against biofilms of Staphylococcus aureus and Staphylococcus epidermidis. Lett Appl Microbiol. 2014;59:507-13.

16. Teplitski M, Al-Agely A, Ahmer BMM. Contribution of the SirA regulon to biofilm formation in Salmonella enterica serovar Typhimurium. Microbiol-Sgm. 2006;152:3411-23.

17. Karunker I, Rotem O, Dori-Bachash M, Jurkevitch E, Sorek R. A global transcriptional switch between the attack and growth forms of Bdellovibrio bacteriovorus. PLoS ONE. 2013;8:e61850.

18. Dwidar M, Im H, Seo JK, Mitchell RJ. Attack-phase Bdellovibrio bacteriovorus responses to extracellular nutrients are analogous to those seen during late intraperiplasmic growth. Microb Ecol. 2017;74:937-46.

19. LaMarre AG, Straley SC, Conti SF. Chemotaxis toward amino acids by Bdellovibrio bacteriovorus. J Bacteriol. 1977;131:201-7.

20. Im H, Kim D, Ghim CM, Mitchell RJ. Shedding light on microbial predator-prey population dynamics using a quantitative bioluminescence assay. Microb Ecol. 2014;67:167-76.

21. Iebba V, Totino V, Santangelo F, Gagliardi A, Ciotoli L, Virga A, et al. Bdellovibrio bacteriovorus directly attacks Pseudomonas aeruginosa and Staphylococcus aureus Cystic fibrosis isolates. Front Microbiol. 2014;5:280. 